\title{
Development of a SYBR-Green I quantitative PCR assay for the detection and genotyping of different hantaviruses
}

\author{
ZIYU LIU $^{1 *}$, FANG WANG $^{1 *}$, LIJUAN YUAN ${ }^{2 *}$, XIAOXIAO ZHANG ${ }^{1}$, QIKANG YING ${ }^{1}$, LAN YU $^{1}$, \\ LIANG ZHANG ${ }^{1}$, LINFENG CHENG ${ }^{1}$, FANGLIN ZHANG ${ }^{1}$, JIANGUO LU $^{2}$ and XING'AN WU ${ }^{1}$ \\ ${ }^{1}$ Department of Microbiology, The Fourth Military Medical University; ${ }^{2}$ Department of General Surgery, \\ Tangdu Hospital, The Fourth Military Medical University, Xi'an, Shaanxi, P.R. China
}

Received December 25, 2015; Accepted June 24, 2016

DOI: $10.3892 /$ ijmm.2016.2678

\begin{abstract}
Hemorrhagic fever with renal syndrome (HFRS) is a severe, viral zoonotic disease which occurs worldwide, particularly in Asia and Europe. In China, the Hantaan virus (HTNV) and the Seoul virus (SEOV) are known to be the most prevalent causative agents of HFRS. Since no protective vaccines or effective treatments are available for human use, accurate and reliable diagnostic methods are essential for disease surveillance. In the present study, the viral loads in cell culture supernatant, infected mice blood and clinical serum samples were quantified using the SYBR-Green I-based reverse transcription-quantitiative polymerase chain reaction (RT-qPCR) assay, which targeted the $\mathrm{S}$ gene sequence of the HTNV and SEOV genomes. The cRNA of these two viruses were synthesized as a positive control and 10 -fold serially diluted from $1 \times 10^{5}$ to $1 \times 10^{0}$ copies $/ \mu 1$. Standard curves were generated by plotting the mean cycle threshold $(\mathrm{Ct})$ values versus copy numbers. The standard curve of HTNV had a correlation coefficient $\left(\mathrm{R}^{2}\right)$ of 0.994 , efficiency of amplification (E) of $101.9 \%$, and the slope of -3.278 , whereas that of SEOV had an $\mathrm{R}^{2}$ of 0.993 , E of $104.8 \%$, and the slope of -3.212 . The minimum detection limit of the RT-qPCR assay for HTNV and SEOV was $10^{1}$ copies/ $\mu$ l. Two qPCR assays were successfully established for the detection of HTNV and SEOV, respectively. Taken together, these findings demonstrate that using the SYBR-Green I-based RT-qPCR assay, the HTNV and SEOV may be genotyped precisely without cross-reactivity. Furthermore, viral RNA may be detected and quantified in cells, mice and
\end{abstract}

Correspondence to: Dr Xing'an Wu, Department of Microbiology, The Fourth Military Medical University, 17 Changle West Road, Xi'an, Shaanxi 710032, P.R. China

E-mail: wuxingan6445@sohu.com

Dr Jianguo Lu, Department of General Surgery, Tangdu Hospital, The Fourth Military Medical University, 169 Xinsi Road, Xi'an, Shaanxi 710038, P.R. China

Email: lujguo@fmmu.edu.cn

*Contributed equally

Key words: Hantaan virus, Seoul virus, hemorrhagic fever with renal syndrome, quantitative PCR, detection, genotyping infected individuals, which may be useful in epidemiological studies as well as for early monitoring and further preventative treatment against SEOV and HTNV-induced diseases.

\section{Introduction}

Hantaviruses belongs to the Bunyaviridae family (1), and cause a serious disease in humans named hemorrhagic fever with renal syndrome (HFRS) which occurs worldwide, particularly in Asia and Europe $(2,3)$. HFRS is one of the most prevalent natural focual infectious diseases in China, and due to the severe symptoms, this virus has the potential to be considered as a bioterrorism agent. As virus transmission to humans occurs by inhalation of the aerosolized virus contained in urine, feces and saliva, by passage through mucous membranes or by bites from infected animals (4), and no protective vaccines or effective treatments are currently available for human use (5), accurate and reliable diagnostic methods are essential for disease surveillance in order to implement adequate public health actions.

Hantaviruses are a group of enveloped, negative sense RNA viruses (6). The genome organization of hantaviruses is typical of other members of the Bunyaviridae family, consisting of three negative-stranded RNA segments with a large (L) segment encoding the viral RNA polymerase, a medium (M) segment encoding the envelope glycoproteins G1 and G2, and a small (S) segment encoding the viral nucleocapsid protein (7).

Thirty genotypes of hantaviruses have been identified worldwide. In China, seven species have been found, but only the Hantaan virus (HTNV) and the Seoul virus (SEOV) have been identified as the most prevalent causative agents of HFRS in this region (6). Serological and genetic analyses showed that HTNV and SEOV co-circulate in rodents (1). It has been reported that disease severity differs among cases of HFRS caused by different hantaviruses. The infection of SEOV usually manifests as a mild form of HFRS and results in lower case fatality rate (8). Compared with the cases caused by SEOV, HFRS caused by HTNV is more severe with a mortality rate of $5-10 \%(2,4)$. Although SEOV-HFRS has a low case fatality rate, complications and long-term hormonal, renal and cardiovascular consequences occur. Therefore, it is important to not only establish a method of diagnosis, but also to detect and distinguish between these two virus species prior to the development of symptoms. 
Many methods have been developed for the diagnosis of hantavirus infections. Common methods used for detection involve immunological techniques (9), such as the immunofluorescence antibody test (IFAT), enzyme-linked immunosorbent assays (ELISA) or immunoblot analysis. Recently, real-time polymerase chain reaction (PCR) assays have also been developed (10,11). SYBR-Green I-based reverse transcription-quantitative polymerase chain reaction (RT-qPCR) has been considered to be technically simpler and more readily available than the TaqMan probe or molecular beacon-based assays $(12,13)$. A recent study has also suggested that there may be an association between the hantavirus RNA load and disease severity (14). Furthermore, it has been suggested that quantifying the load of infectious virions was essential for the effective treatment of HTNV infection (15).

In the present study, the viral loads in cell culture supernatant, infected mice blood and clinical serum samples were quantified by SYBR-Green I-based RT-qPCR, which targeted the $\mathrm{S}$ gene sequence of the HTNV and SEOV genomes. The results obtained from two different viruses (HTNV and SEOV) were compared. The viral RNA levels were also evaluated using serum samples obtained from infected mice at different time points post infection.

\section{Materials and methods}

Virus, cells and mice. The virus strains used in this study were the HTNV strain 76-118 (from our laboratory) and the SEOV strain SR-11 (a gift from Anhui Medical University, Hefei, China). Vero E6 cells (ATCC CRL-1586) were cultured in RPMI-1640 (Gibco, Grand Island, NY, USA) supplemented with L-glutamine and $10 \%$ fetal calf serum (FCS). Sixty BALB/c mice (3-4-days old) were obtained from the Animal Research Centre at the Fourth Military Medical University (Xi'an, China).

Design of specific primers. The primers used in this study were designed using Oligo 6.0 software by multiplying aligning $\mathrm{S}$ segment nucleotide sequences from different strains of HTNV and SEOV viruses, respectively. The following primer pairs were used: HTNV forward, 5'-GAG CCT GGA GAC CAT CTG-3' and reverse, 5'-CGG GAC GAC AAA GGA TGT-3' (the product was $144 \mathrm{bp}$ ); SEOV forward, 5'-GGG AAT ACA CTG GAC CTG-3' and reverse, 5'-GTC CTT TGA AGT CTG CCT-3' (the product was $159 \mathrm{bp}$ ). The primers were synthesized by Augct DNA-Syn Biotechnology Co., Ltd., (Beijing, China).

RNA extraction. Total RNA from virus-infected cell cultures and mice serum were extracted using the RNAfast1000 kit (Pioneer Biotech Co., Ltd., Xi'an, China). The RNA from human serum samples were extracted using the QIAamp viral RNA mini kit (Qiagen, Hilden, Germany) according to the manufacturer's instructions. The RNA was recovered in $20 \mu \mathrm{l}$ nuclease-free water and stored at $-80^{\circ} \mathrm{C}$.

Preparation of cRNA standard. To prepare the HTNV and SEOV cRNA standard for the quantitative PCR assay, total RNA was extracted from cells infected with HTNV and SEOV, respectively. The cDNA was derived from the highly conserved
S gene sequence from the HTNV and SEOV genomes, respectively. One hundred units of SuperScript II reverse transcriptase was used in $1 \mathrm{X}$ First-Strand Buffer (Invitrogen, Carlsbad, CA, USA) at $20^{\circ} \mathrm{C}$ for $10 \mathrm{~min}, 42^{\circ} \mathrm{C}$ for $90 \mathrm{~min}, 95^{\circ} \mathrm{C}$ for $5 \mathrm{~min}$ and the reaction was cooled to $4^{\circ} \mathrm{C}$. The cDNA ( 1.3 kb in length) were amplified by PCR and cloned into the pMD18T vector (Takara, Dalian, China). The HTNV S gene sequence in the plasmid was named HTNV-pMD18T-S and the SEOV S gene sequence in the plasmid was named SEOV-pMD18T-S. They were confirmed by PCR and subsequent DNA sequencing. Plasmid isolation was performed using Axygen Plasmid Mini-Prep kits (Axygen, Union City, CA, USA). Subsequently, the cRNA was synthesized by in vitro transcription using T7 RNA polymerase (Takara Biotechnology, Dalian, China) in accordance with the manufacturer's instructions. The cRNA was processed twice with DNase I (Takara Biotechnology), then the residues were precipitated using chloroform/isoamyl alcohol and alcohol/ethanol. The cRNA was finally dissolved in $50 \mu \mathrm{l}$ RNase-free water and electrophoresed in a $2 \%$ agarose gel (Biowest, Madrid, Spain). DL3000 DNA Marker (Proteri Biotechnology Co., Ltd., Dalian, China) was used as molecular weight marker. The cRNA was stored at $-80^{\circ} \mathrm{C}$.

The cRNA were assessed for purity using the A260/280 ratio and the concentration was determined from A260 using the spectrophotometer (6405UV; Jenway, Burlington, NJ, USA) to produce a best-fit linear regression of the standard curve. The standards were run in triplicate.

Quantitative PCR. The quantitative PCR step was performed using SYBR ${ }^{\circledR}$ Premix Ex Taq ${ }^{\mathrm{TM}}$ (Takara) on a Stratagene MX3005P real-time qPCR system (Agilent Technologies, Inc., Santa Clara, CA, USA). The standard cycling conditions were as follows: $95^{\circ} \mathrm{C}$ for $10 \mathrm{sec}$, followed by 40 cycles of $95^{\circ} \mathrm{C}$ for $5 \mathrm{sec}, 60^{\circ} \mathrm{C}$ for $20 \mathrm{sec}$ and $72^{\circ} \mathrm{C}$ for $1 \mathrm{~min}$. Quantitative PCR reaction components were set-up in triplicate according to the manufacturer's instructions. The cycle threshold $(\mathrm{Ct})$ for each gene was determined by setting the $\mathrm{Ct}$ line at the center of the logarithmic phase of amplification for that particular amplicon.

Generation of standard curves using quantitative PCR. The cRNA standards were 10 -fold serial diluted over range from $1.0 \times 10^{5}$ to $1.0 \times 10^{0}$ copies $/ \mu 1$ using Easy Dilution (Takara Biotechnology). The mean values were obtained by testing in triplicate. The standard curves were generated by plotting $\mathrm{Ct}$ values versus the log copy numbers. Regression analysis, standard curve slopes and amplification efficiencies were calculated using automated software (Stratagene MX3005P qPCR software).

Specificity and sensitivity. The specificity of the assay was assessed by processing two different templates from HTNV and SEOV during RT-qPCR. Additionally, the total cellular RNA extracts were tested and melting curve analysis of PCR products was performed in order to exclude the presence of unspecific products or primer dimer synthesis. Serial dilutions in the range of $1.0 \times 10^{5}$ to $1.0 \times 10^{0}$ copies $/ \mu$ cRNA standards were used in triplicate to determine the sensitivity of the assay. To calculate the corresponding number of RNA molecules, a cRNA reference standard curve was processed in parallel by qPCR. 

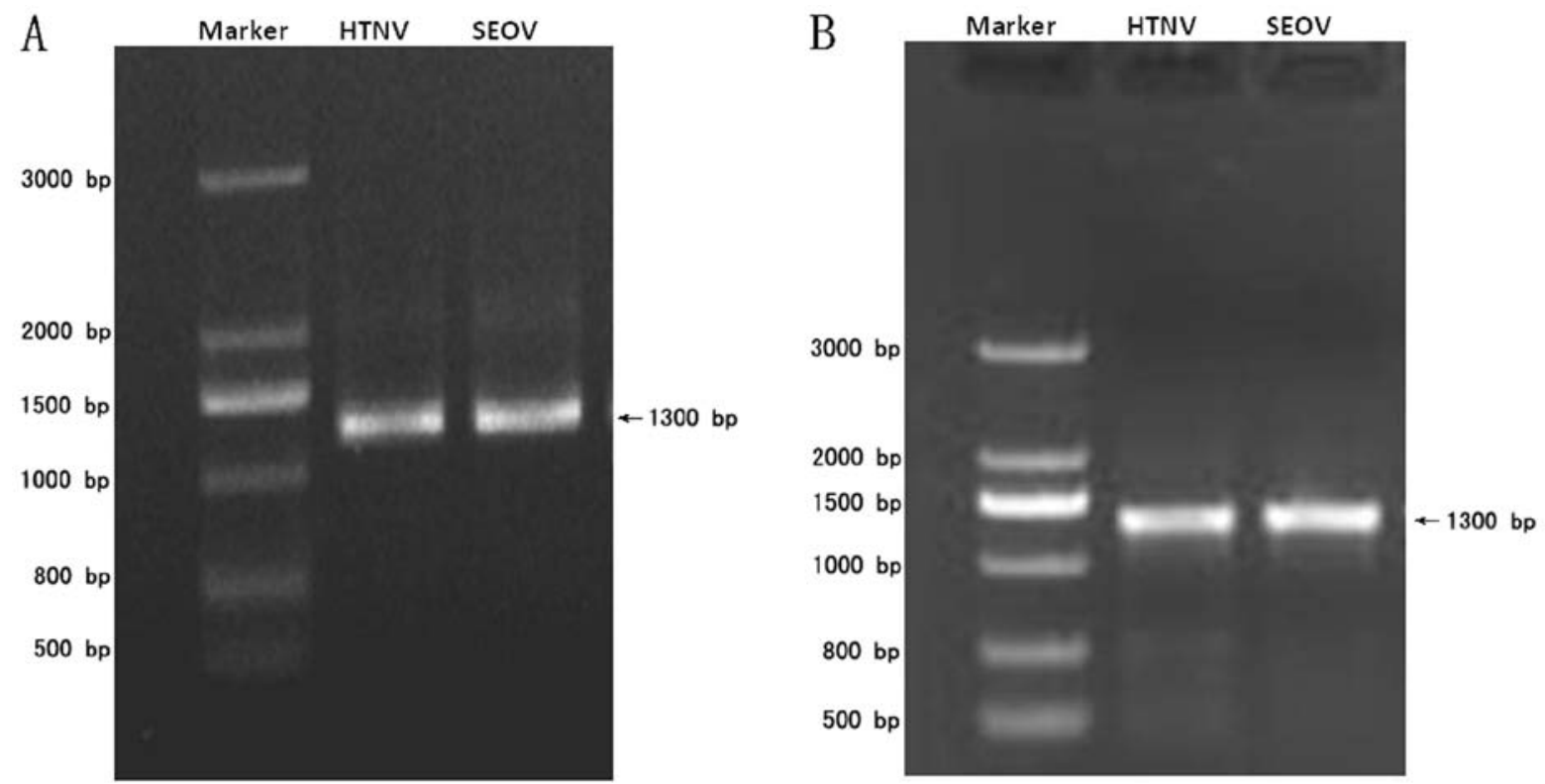

Figure 1. Identification of Hantavirus cDNA and cRNA. (A) Agarose gel electrophoresis of Hantaan virus (HTNV) and Seoul virus (SEOV) S gene cDNA of plasmid pMD18T-S, respectively. Marker: DL3000 DNA Marker (B) Agarose gel electrophoresis of cRNA of both HTNV and SEOV. Marker: DL3000 DNA Marker. cDNA with a length of $1.3 \mathrm{~kb}$ was synthesized by reverse transcription and subsequently amplified by PCR. Hantavirus cRNA with a length of $1.3 \mathrm{~kb}$ was synthesized using the cDNA as template by in vitro transcription.

Mice infected with hantavirus. Suckling mice were infected with viral suspensions $\left(10^{5} \mathrm{pfu}\right)$ of HTNV and SEOV, respectively. Any death occurring $24 \mathrm{~h}$ post infection (p.i.) was considered as a traumatic injury and was excluded from the following experiment. Whole blood, was extracted and left to stand for $1 \mathrm{~h}$, and centrifuged at 3,000 rpm for $15 \mathrm{~min}$. The serum was obtained from the supernatant. RNA was extracted from the serum of the mice, obtained on different days. The study received ethics approval from the Ethics Committee of the Forth Military Medical University (Xi'an, China). The mice infected with HTNV died from the disease onset, and the mice infected with SEOV were sacrificed when the experiment ended.

Clinical specimens. Thirteen patients diagnosed with HFRS and 2 healthy individuals (normal controls) at the Department of Infectious Diseases at Tangdu Hospital (Xi'an, China) were enrolled in this study in 2013. Peripheral blood was collected during the febrile phase of the illness (3-7 days after the onset of fever) and the extracted serum was stored at $-80^{\circ} \mathrm{C}$. The diagnosis of HFRS was confirmed by the detection of hantavirus IgM/IgG antibodies according to the manufacturer's instructions for Hantavirus IgG/IgM Combo Test Card (Boson Biotech, Xiamen, China). The study received ethics approval from the Ethics Committee of Tangdu Hospital (Xi'an, China) and written informed consent was obtained from all enrolled subjects.

Statistical analysis. All analyses were performed using Excel and SPSS version 13.0 software. Data were analyzed using a $\chi^{2}$-test.

\section{Results}

Preparation of the positive controls and synthesis of the $c R N A$. The S gene cDNA for either HTNV or SEOV were inserted into the pMD18T vector. The successful construc- tion of both recombinant plasmids was confirmed by DNA sequencing. The expected fragments of $\sim 1.3 \mathrm{~kb}$ were obtained by PCR, respectively (Fig. 1A). The cRNA were synthesized in vitro using T7 RNA polymerase and the cDNA as templates. Two cRNAs of $\sim 1.3 \mathrm{~kb}$ were obtained, respectively (Fig. 1B). The copy number of cRNA was calculated according to the concentration; HTNV and SEOV cRNA were diluted to $1 \times 10^{5}$ copies $/ \mu 1$, respectively.

Standardization of SYBR-Green I-based RT-qPCR. Standard curves for the detection of HTNV and SEOV RNA by SYBR-Green I-based RT-qPCR were established. The thermal profile of the PCR were optimized at an annealing temperature of $60^{\circ} \mathrm{C}$. A primer concentration of $0.4 \mu \mathrm{M}$ in $25 \mu \mathrm{l}$ was used, in a reaction scale. The RNA standards were both diluted by 10 -fold serial dilutions ranging from $1 \times 10^{5}$ to $1 \times 10^{0}$ copies $/ \mu 1$, and the standard curves were generated by plotting $\mathrm{Ct}$ versus the copy number in the amplification chart (Fig. 2). The mean values were linear over the entire range of HTNV cRNA dilutions with a correlation coeeficient $\left(\mathrm{R}^{2}\right)$ of 0.994 , efficiency of amplification (E) of $101.9 \%$, and the slope of -3.278 (Fig. 2A and B). Moreover, linearity was good for the standard curve of SEOV cRNA dilutions, with $\mathrm{R}^{2}$ of 0.993 , E of $104.8 \%$, and the slope of -3.212 (Fig. 2D and E). The gradients of the lines were 2-3 Ct/10-fold concentration change.

Sensitivity and specificity of SYBR-Green I-based RT-qPCR. The standards were diluted by 10 -fold serial dilutions ranging from $1 \times 10^{5}$ to $1 \times 10^{0}$ copies $/ \mu 1$. According to the established assay, the minimum detection limit of RT-qPCR for HTNV and SEOV was $10^{1}$ copies $/ \mu 1$. Furthermore, SYBR-Green I-based RT-qPCR was highly specific for HTNV and SEOV, respectively (Fig. 2C and F). No primer dimers or non-specific products were found by melting curve analysis. Only one single sharp peak was visible in both the melt peak charts. 

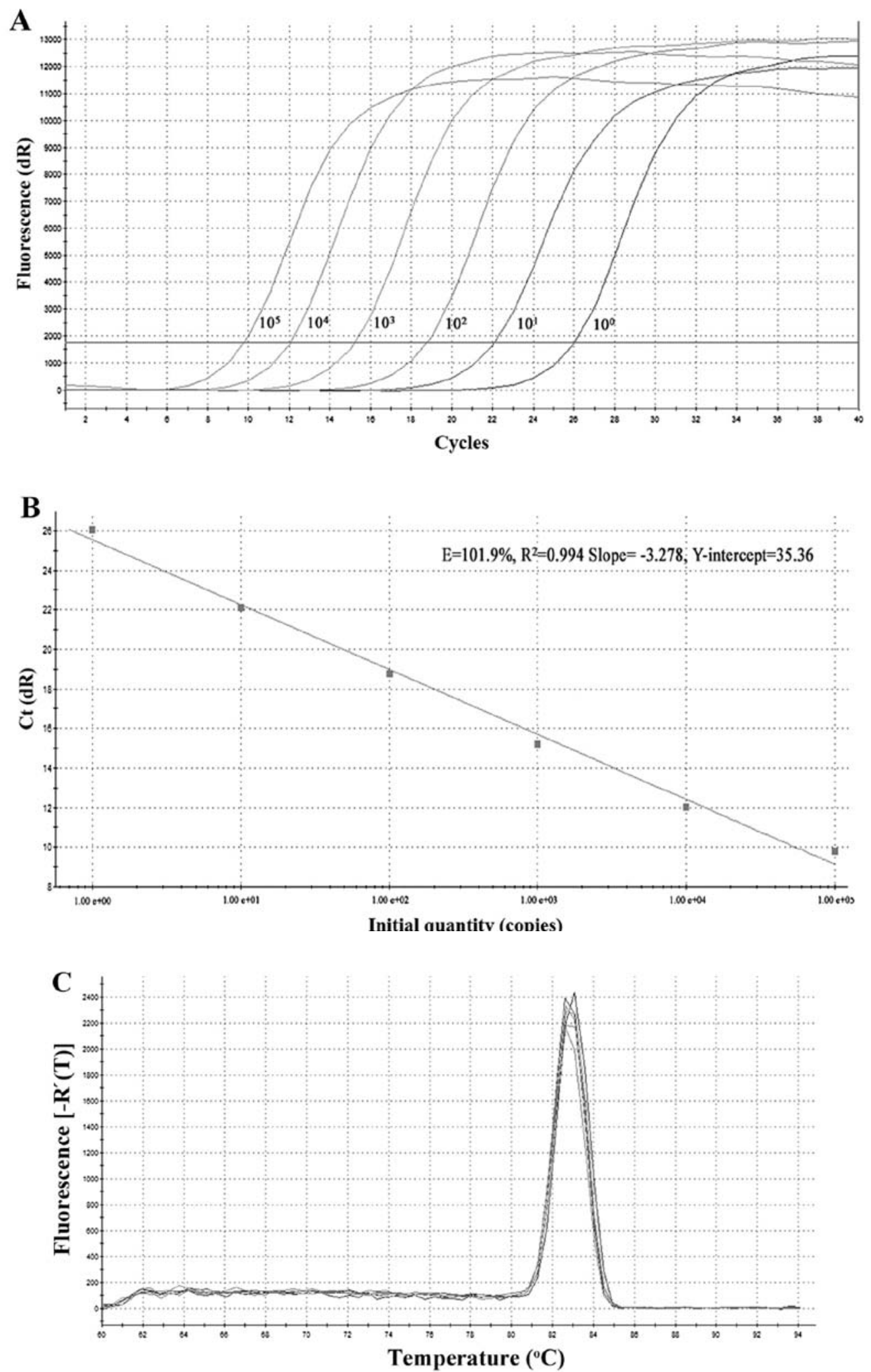

Figure 2. SYBR-Green I-based RT-qPCR amplification chart and standard curve derived from serially diluted standard cRNA. (A) Amplification plots for 10 -fold serial dilutions of the Hantaan virus (HTNV) cRNA standards ranging from $1.0 \times 10^{5}$ to $1.0 \times 10^{0}$ copies $/ \mu 1$. (B) The HTNV cRNA standard curve was plotted by mean Ct value vs. the HTNV cRNA log copy numbers. (C) Melting peaks of the PCR products obtained from 10-fold serial dilutions of the HTNV cRNA standards. Only a single sharp peak at $83^{\circ} \mathrm{C}$ was visible in the melt peak chart. $\mathrm{R}^{2}$, correlation coefficient; E, efficiency of amplification.

Genotyping of hantaviruses from infected cells. The Vero E6 cells were infected with HTNV and SEOV, respectively. There was no evidence of the virus cytopathic effect (CPE) following infection with either of these two viruses. The viral RNA was collected from day 4. SYBR-Green I-based RT-qPCR was conducted according to the previously established assay. Both $\mathrm{Ct}$ values of the amplification curve were obtained. No cross-reactivity was observed between the 

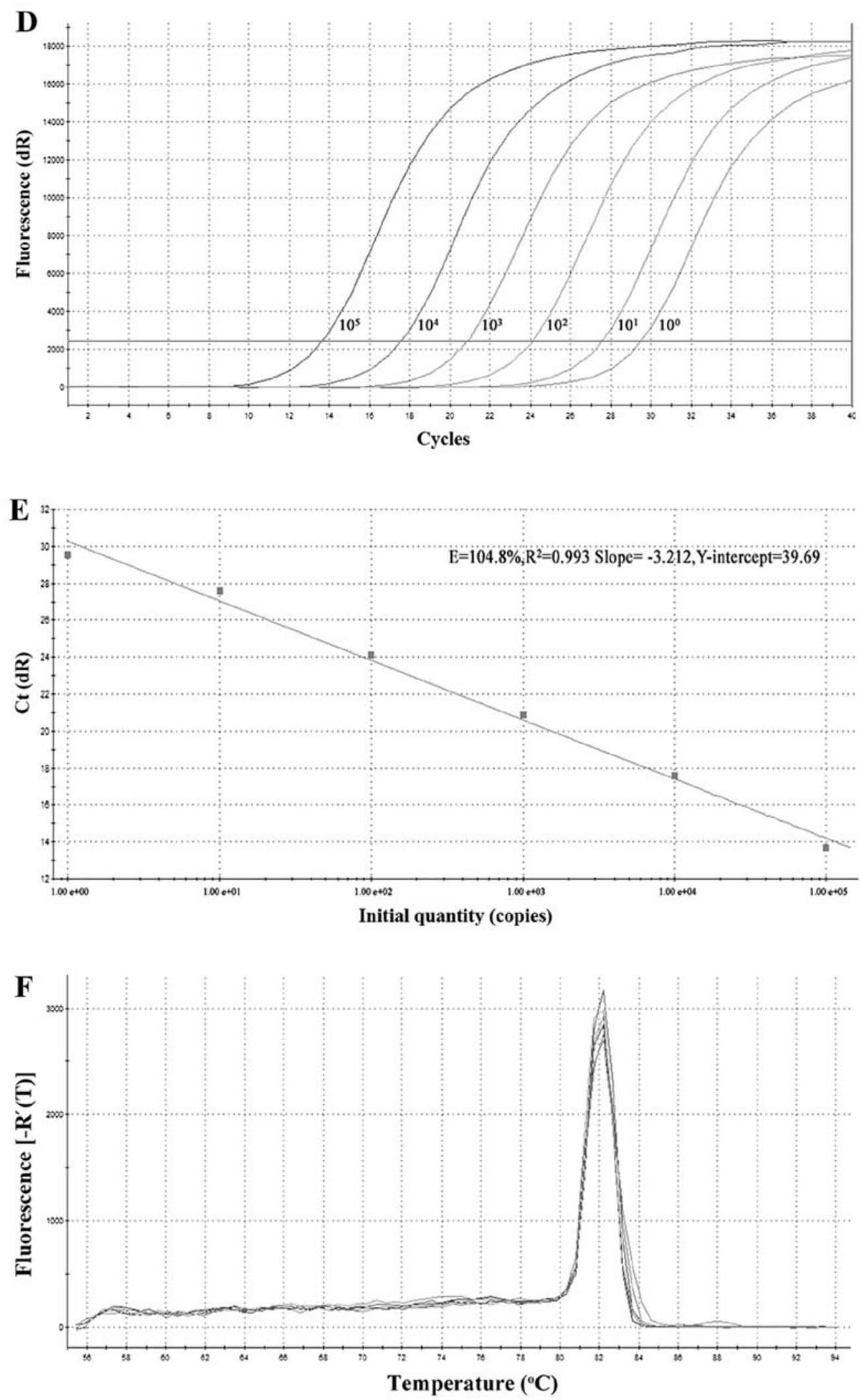

Figure 2. Continued. (D) Amplification plots for 10-fold serial dilutions of the Seoul virus (SEOV) cRNA standards ranging from 1.0x10 10 to $1.0 \times 10^{\circ} \mathrm{copies} / \mu 1$. (E) The SEOV cRNA standard curve was plotted by mean Ct value vs. the SEOV cRNA log copy numbers. (F) Melting peaks of the PCR products obtained from 10 -fold serial dilutions of the SEOV cRNA standards. Only a single sharp peak at $82^{\circ} \mathrm{C}$ was visible in the melt peak chart. $\mathrm{R}^{2}$, correlation coefficient; E, efficiency of amplification.

HTNV and SEOV (Fig. 3A and C). Negative amplification were obtained and melting curve analysis showed no sharp peak on the melting curve chart when using the improper primers (Fig. 3B and D).
Assay performance on samples from infected mice. In the next experiment, suckling mice were infected with HTNV and SEOV, respectively. As shown in Fig. 4, the special amplification curve (the $\mathrm{Ct}$ value for HTNV was 25-34) appeared from 

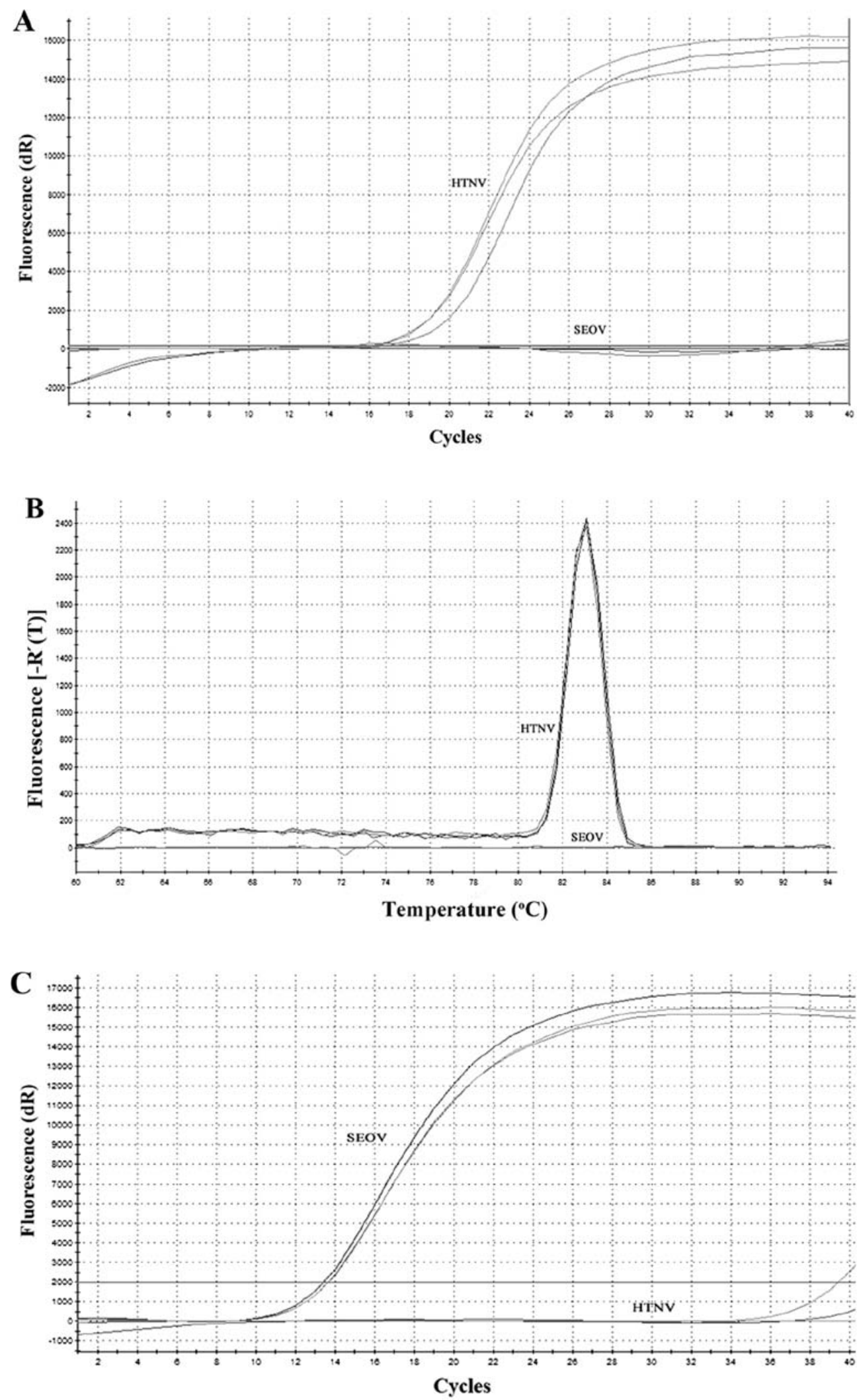

Figure 3. Genotyping of hantavirus using SYBR-Green I-based RT-qPCR. (A) Hantaan virus (HTNV) RNA from infected cells was amplified by SYBR-Green I-based RT-qPCR using two primer pairs, respectively. Only HTNV RNA was amplified.(B) A single sharp peak was observed in the melting chart indicated that only specific products of HTNV RNA was obtained. (C) Seoul virus (SEOV) RNA from infected cells was amplified by SYBR-Green I-based RT-qPCR using two primer pairs, respectively. Only SEOV RNA was amplified.

day 2 to 10 after infection (Fig. 4A and B), which demonstrated that HTNV RNA is detectable on the second day after infection. Furthermore, the load of HTNV RNA increased with persistent infection of virus until disease onset. The viral load stablized on the sixth day. Compared with HTNV infection, SEOV infection was not lethal. The Ct value of SEOV decreased post infection; 


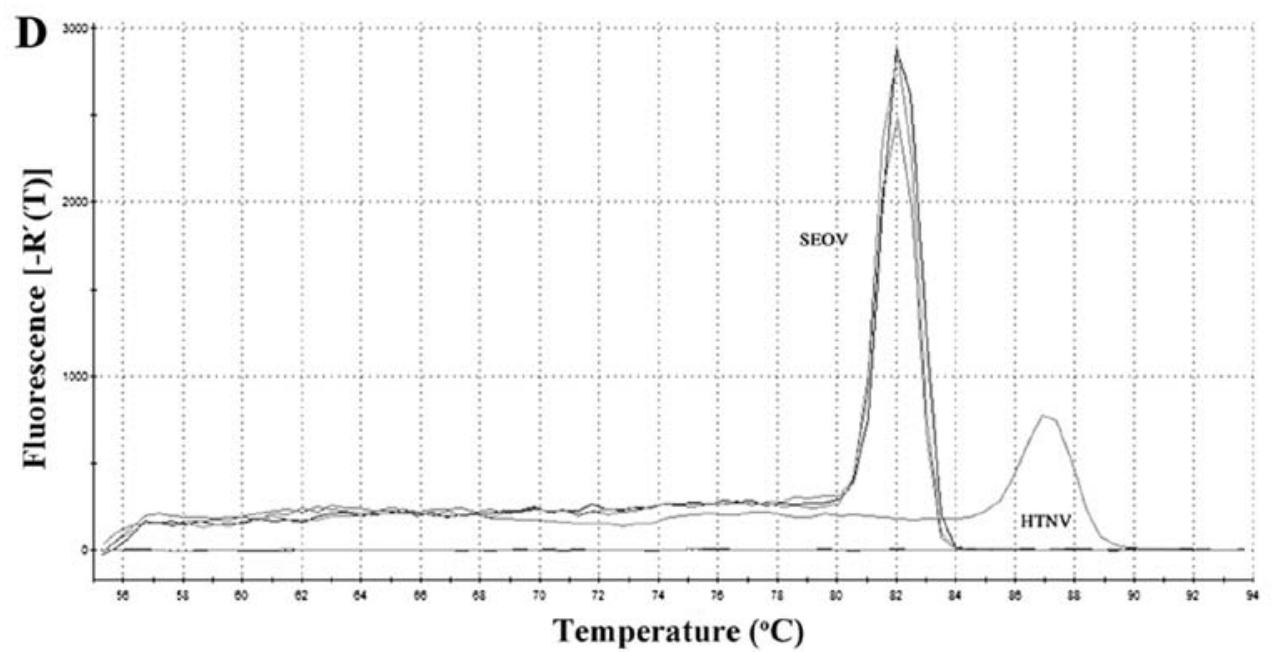

Figure 3. Continued. (D) A single sharp peak was observed in the melting chart which indicated that only specific products of SEOV RNA were obtained.
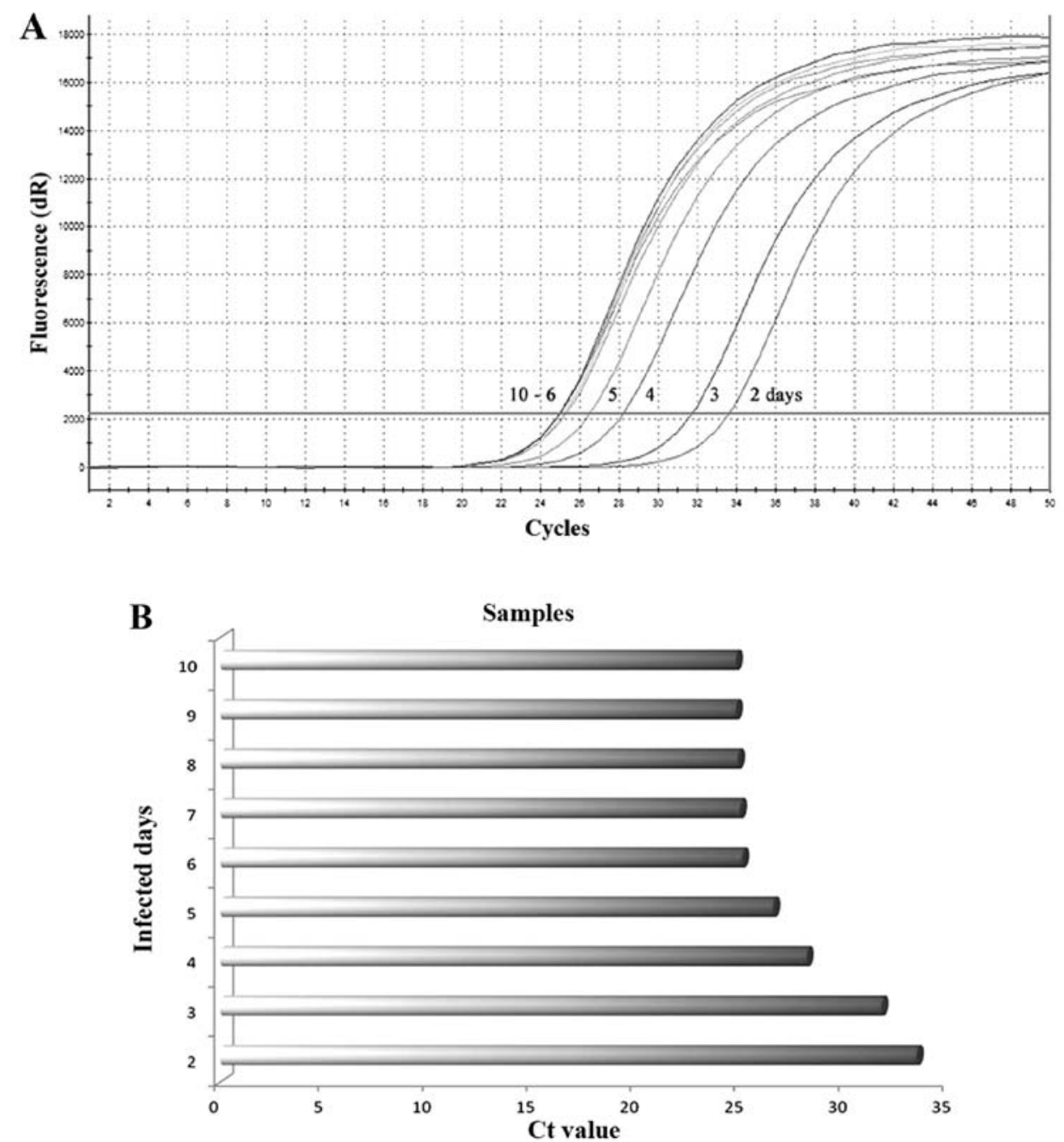

Figure 4. Variations in RNA copy number in the serum from mice infected by hantavirus on different days post infection. (A and B) RT-qPCR results using serum from mice infected by Hantaan virus (HTNV), extracted on day 2 to 10. The HTNV RNA copy number increased until day 6 and then stablized.

however, it started increasing after reaching its lowest level on day 16 (Fig. 4C and D). This was consistent with the study that SEOV infection would not result in exposure.
Assay performance on clinical samples. In order to evaluate the usefulness of the SYBR-Green I-based RT-qPCR assay established herein, serum samples from 13 HFRS patients and 

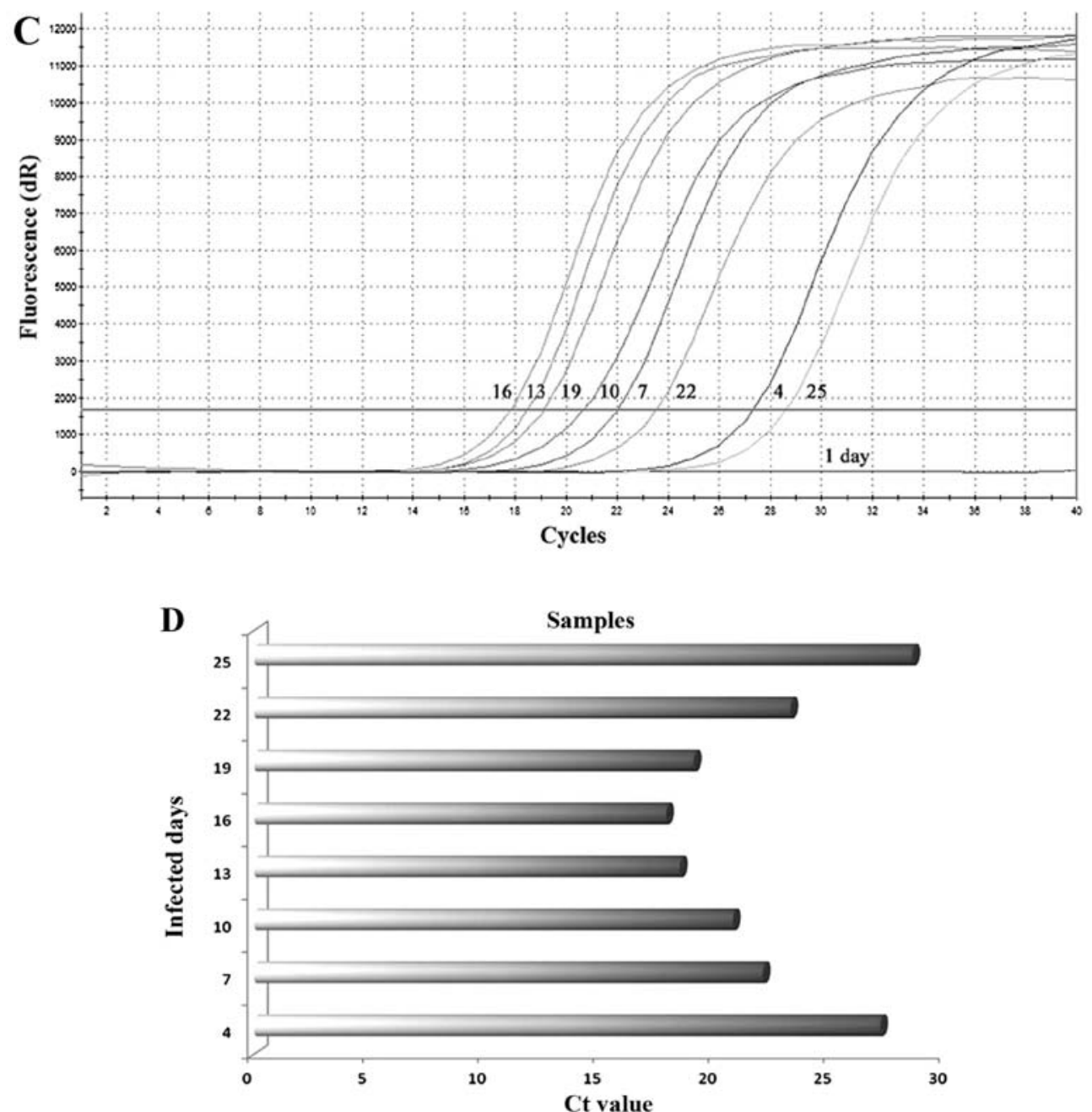

Figure 4. Continued. (C and D) RT-qPCR results using serum from mice infected by Seoul virus (SEOV) on day 1 to 25 post infection. The SEOV RNA copy number increased after infection, but it decreased after reaching a peak on day 16.

Table I. Quantification of HTNV RNA in serum samples obtained from patients and normal controls using the SYBRGreen I-based RTq-PCR assay.

\begin{tabular}{lccccc}
\hline Sample no. & $\begin{array}{c}\text { HTNV-IgM/IgG } \\
\text { antibodies Combo test }\end{array}$ & $\begin{array}{c}\text { Days after onset } \\
\text { of fever }\end{array}$ & $\begin{array}{c}\text { Severity of the } \\
\text { HFRS disease }\end{array}$ & $\begin{array}{c}\text { Copies/ } \mu 1 \text { of the } \\
\text { HTNV RNA }\end{array}$ & $\begin{array}{c}\text { Copies/ } \mu \text { of the } \\
\text { SEOV RNA }\end{array}$ \\
\hline HFRS 1 & Positive/positive & 5 & Severe & $7.53 \times 10^{6}$ & $\begin{array}{c}\text { Negative } \\
\text { HFRS 2 }\end{array}$ \\
Positive/positive & 7 & Critical & $7.53 \times 10^{6}$ & Negative \\
HFRS 3 & Positive/positive & 5 & Critical & $8.67 \times 10^{5}$ & Negative \\
HFRS 4 & Positive/positive & 6 & Critical & $5.75 \times 10^{5}$ & Negative \\
HFRS 5 & Positive/positive & 4 & Severe & $9.32 \times 10^{4}$ & Negative \\
HFRS 6 & Positive/negative & 6 & Moderate & $1.34 \times 10^{4}$ & Negative \\
HFRS 7 & Positive/positive & 5 & Severe & $8.55 \times 10^{3}$ & Negative \\
HFRS 8 & Positive/positive & 4 & Severe & $4.15 \times 10^{3}$ & Negative \\
HFRS 9 & Positive/negative & 3 & Mild & $2.86 \times 10^{3}$ & Negative \\
HFRS 10 & Positive/positive & 5 & Moderate & $5.63 \times 10^{2}$ & Negative \\
HFRS 11 & Positive/positive & 3 & Moderate & $4.78 \times 10^{2}$ & Negative \\
HFRS 12 & Positive/negative & 4 & Mild & $3.74 \times 10^{2}$ & Negative \\
HFRS 13 & Positive/positive & 3 & Mild & $2.42 \times 10^{2}$ & - \\
Normal 1 & $-/-$ & - & - & - & - \\
Normal 2 & $-/-$ & - & - & - & - \\
\hline
\end{tabular}

${ }^{a}$ The severity of HFRS disease was classified according to the clinical symptoms and laboratory parameters used in the diagnostic criteria for HFRS in China as mild, moderate, severe, and critical. HTNV, Hantaan virus; SEOV, Seoul virus; HFRS, hemorrhagic fever with renal syndrome. 

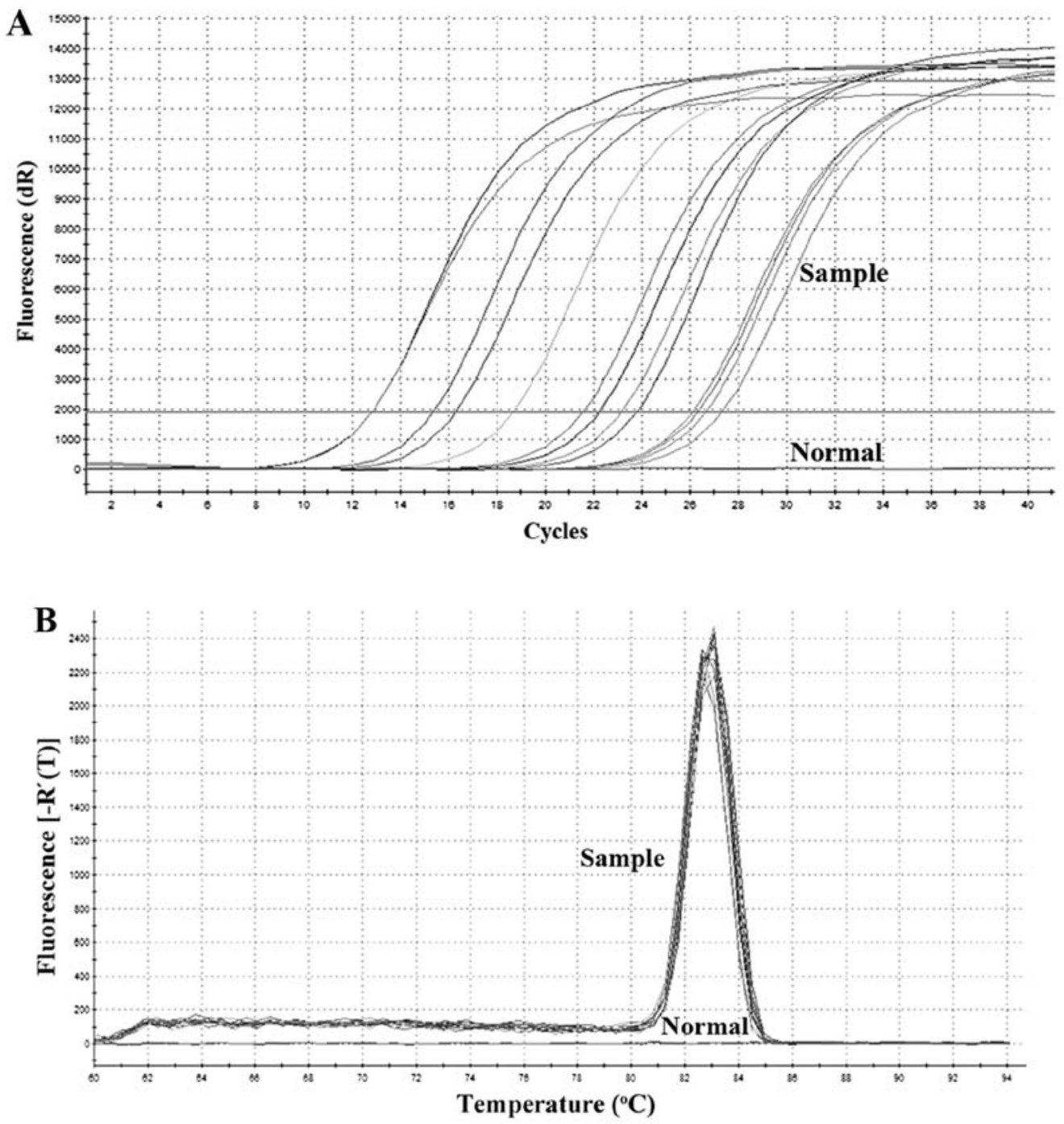

Figure 5. SYBR-Green I-based RT-qPCR results using clinical samples of serum obtained from 13 patients diagnosed with acute hemorrhagic fever with renal syndrome (HFRS) and 2 normal controls. (A) Diagnosis of clinical samples using Hantaan virus (HTNV) primers. The copy number of the HTNV RNA ranged from $2.42 \times 10^{2}$ to $7.53 \times 10^{6}$. (B) Melting chart of the PCR products obtained from the clinical samples. A single sharp peak was observed in the melting chart which indicated that only specific products of HTNV RNA were obtained.

two normal controls were collected for analysis. All the HFRS patients had been diagnosed from clinical symptoms and laboratory examinations. Peripheral blood was collected during the febrile phase of the illness (3-7 days after the onset of fever). As shown in Fig. 5, no positive results were obtained from the normal control samples; however, the copy numbers of HTNV RNA in the serum samples from HFRS patients ranged from $2.42 \times 10^{2}$ to $7.53 \times 10^{6}$. Negative results were obtained when the samples were analyzed using SEOV primers (Table I).

\section{Discussion}

HFRS caused by hantavirus infection has been considered to be a serious and often fatal disease, with approximately $90 \%$ of all cases reported in China $(7,16)$. The genotypes of hantavirus causing HFRS in mainland China are mainly the HTNV and the SEOV. Disease caused by HTNV is more severe clinically, whereas SEOV infection results in a longer incubation period and sometimes, may not lead to the onset of HFRS. Nucleic acid-based methods, such as RT-PCR, are sensitive and allow for species-specific virus identification. More recently, RT-qPCR was used to identify Puumala hantavirus (PUUV) (11) as well as other hemorrhagic fevercausing agents such as Ebola, Marburg, Lassa, Crimean-Congo haemorrhagic fever (CCHF), dengue, Rift Valley fever (RVF) and Yellow fever virus (YFV) (17-19). In the present study, the RT-qPCR assay was successfully established for the detection of the HTNV and SEOV. By means of SYBR-Green I-based RT-qPCR, the HTNV and SEOV can be genotyped precisely without cross-reactivity. Furthermore, individuals infected with SEOV may be diagnosed which is of value in the prevention and treatment of SEOV-induced disease.

In real-time PCR, the synthesis of the amplification product is monitored during the reaction allowing quantification of the viral RNA in the sample. RT-qPCR has been proved to have exceptional analytical sensitivity and specificity $(13,15)$. In this study, we have established a quantitative PCR (qPCR) assay for HTNV and SEOV using cRNAs generated by the plasmid pMD18T-S, which contained the $S$ gene sequence of these two viruses. Standard curves of HTNV and SEOV were 
described with excellent linear relationships over a range of $1 \times 10^{5}$ to $1 \times 10^{0}$ copies $/ \mu 1$ cRNA with $\mathrm{R}^{2}=0.994$ and $\mathrm{R}^{2}=0.993$, respectively. The limit of sensitivity was $10^{1} \mathrm{cRNA}$ copies $/ \mu 1$. High E values for HTNV and SEOV were shown as 101.9 and $104.8 \%$, respectively. These properties of the established SYBR-Green I-based RT-qPCR assay showed an improved detection limit and that the test is ideal for use with animal models as well as in clinical practice.

A major disadvantage of SYBR-Green I-based RT-qPCR is that SYBR-Green dyes intercalate into any double-stranded DNA including primer-dimers and non-specific amplification products. Therefore, well designed primers and melt curve analysis is required in the SYBR-Green based assay for the discrimination of specific products from by-products $(20,21)$. In addition, the optimization of annealing temperatures and primer concentrations were crucial in preventing the non-specific amplification of other unrelated gene products. The primers for the S gene sequence of both HTNV and SEOV are well designed and strictly verified, the reaction assays were optimized and only one sharp peak showed in each melt curve analysis, indicating quite good sensitivity and specificity of the assay and the absence of by-products. Furthermore, the risk of contamination is greatly reduced as the PCR product is detected within a closed tube.

Viral RNA from different specimens of infected individuals is detectable, preferentially during the acute phase of hantavirus infection or even prior to the signs of infection becoming apparent. The methods were also applied in the evaluation of viral load in samples from infected mice prior to disease onset. Elevated levels of viral RNA have been recorded in mice suffering from HTNV infection until the onset of disease. However, in the present study, the level of viral RNA in the serum of mice infected with SEOV rose first, and then fell and failed to induce the onset of disease, which is consistent with the findings of previous experiments performed at our laboratory (data unpublished), that SEOV almost never induces HFRS. Therefore, patients infected with SEOV were not included herein for analysis. Although the pathogenesis of hantavirus infection remains unclear, these findings indicated that the load and replication of viruses played a pivotal role in the pathogenesis of hantavirus infection in suckling mice and potentially during hantavirus infection in humans based on the results of the RT-qPCR assay.

This study presents the use of the established SYBR-Green I-based RT-qPCR assay and specific primers for the evaluation of HTNV and SEOV RNA in vitro and in vivo. This method proved to be highly sensitive, specific and reproducible in cRNA positive controls, infected cells, infected mice and clinical specimens. The low cost and rapid nature of the technique suggest that this is a useful new tool for monitoring and evaluating variations in viral RNA levels. The clinical application of this method may also prove invaluable to clinicians and public health officials who need to detect this important pathogen in individuals affected by natural infection or man-made exposure (bioterrorism).

\section{Acknowledgements}

The present study was supported by the National Natural Science Foundation of China (grant nos. 31270978 and 31470890). It was finished at the Department of Microbiology of Fourth Military Medical University. The authors would like to thank all the staff at Department of Infectious Disease of Tangdu Hospital for their support of this study.

\section{References}

1. Schmaljohn CS, Hasty SE, Harrison SA and Dalrymple JM: Characterization of Hantaan virions, the prototype virus of hemorrhagic fever with renal syndrome. J Infect Dis 148: 1005-1012, 1983

2. Jonsson CB, Figueiredo LT and Vapalahti O: A global perspective on hantavirus ecology, epidemiology, and disease. Clin Microbiol Rev 23: 412-441, 2010.

3. Vaheri A, Henttonen H, Voutilainen L, Mustonen J, Sironen T and Vapalahti O: Hantavirus infections in Europe and their impact on public health. Rev Med Virol 23: 35-49, 2013.

4. Roda Gracia J, Schumann B and Seidler A: Climate variability and the occurrence of human puumala hantavirus infections in Europe: a systematic review. Zoonoses Public Health 62: 465-478, 2015.

5. Maes P, Clement J, Gavrilovskaya I and Van Ranst M: Hantaviruses: Immunology, treatment, and prevention. Viral Immunol 17: 481-497, 2004.

6. Kanerva M, Mustonen J and Vaheri A: Pathogenesis of puumala and other hantavirus infections. Rev Med Virol 8: 67-86, 1998.

7. Khaiboullina SF, Morzunov SP and St Jeor SC: Hantaviruses: molecular biology, evolution and pathogenesis. Curr Mol Med 5: 773-790, 2005.

8. Clement J, Heyman P, McKenna P, Colson P and Avsic-Zupanc T: The hantaviruses of Europe: from the bedside to the bench. Emerg Infect Dis 3: 205-211, 1997.

9. Hedman K, Vaheri A and Brummer-Korvenkontio M: Rapid diagnosis of hantavirus disease with an IgG-avidity assay. Lancet 338: 1353-1356, 1991.

10. Mohamed N, Nilsson E, Johansson P, Klingström J, Evander M, Ahlm C and Bucht G: Development and evaluation of a broad reacting SYBR-green based quantitative real-time PCR for the detection of different hantaviruses. J Clin Virol 56: 280-285, 2013.

11. Garin D, Peyrefitte C, Crance JM, Le Faou A, Jouan A and Bouloy M: Highly sensitive Taqman PCR detection of Puumala hantavirus. Microbes Infect 3: 739-745, 2001.

12. Dash PK, Boutonnier A, Prina E, Sharma S and Reiter P: Development of a SYBR-Green I based RT-PCR assay for yellow fever virus: application in assessment of YFV infection in Aedes aegypti. Virol J 9: 27, 2012.

13. Jiang W, Yu HT, Zhao K, Zhang Y, Du H, Wang PZ and Bai XF: Quantification of Hantaan virus with a SYBR-Green I-based one-step qRT-PCR assay. PLoS One 8: e81525, 2013.

14. Yi J, Xu Z, Zhuang R, Wang J, Zhang Y, Ma Y, Liu B, Zhang Y, Zhang $\mathrm{C}$, Yan $\mathrm{G}$, et al: Hantaan virus RNA load in patients having hemorrhagic fever with renal syndrome: correlation with disease severity. J Infect Dis 207: 1457-1461, 2013.

15. Wei F, Li JL, Ling JX, Chen LJ, Li N, Liu YY, Luo F, Xiong HR, Hou W and Yang ZQ: Establishment of SYBR-Green-based qPCR assay for rapid evaluation and quantification for anti-Hantaan virus compounds in vitro and in suckling mice. Virus Genes 46 : 54-62, 2013.

16. Schmaljohn $\mathrm{C}$ and Hjelle B: Hantaviruses: a global disease problem. Emerg Infect Dis 3: 95-104, 1997.

17. Bird BH, Bawiec DA, Ksiazek TG, Shoemaker TR and Nichol ST: Highly sensitive and broadly reactive quantitative reverse transcription-PCR assay for high-throughput detection of Rift Valley fever virus. J Clin Microbiol 45: 3506-3513, 2007.

18. Sall AA, Macondo EA, Sène OK, Diagne M, Sylla R, Mondo M, Girault L, Marrama L, Spiegel A, Diallo M, et al: Use of reverse transcriptase PCR in early diagnosis of Rift Valley fever. Clin Diagn Lab Immunol 9: 713-715, 2002.

19. Drosten C, Göttig S, Schilling S, Asper M, Panning M, Schmitz H and Günther S: Rapid detection and quantification of RNA of Ebola and Marburg viruses, Lassa virus, Crimean-Congo hemorrhagic fever virus, Rift Valley fever virus, dengue virus, and yellow fever virus by real-time reverse transcription-PCR. J Clin Microbiol 40: 2323-2330, 2002.

20. Jiang W, Wang PZ, Yu HT, Zhang Y, Zhao K, Du H and Bai XF: Development of a SYBR-Green I based one-step real-time PCR assay for the detection of Hantaan virus. J Virol Methods 196: 145-151, 2014.

21. Chen H, Parimelalagan M, Lai YL, Lee KS, Koay ES, Hapuarachchi HC, Ng LC, Ho PS and Chu JJ: Development and evaluation of a SYBR-Green-based real-time multiplex RT-PCR assay for simultaneous detection and serotyping of dengue and Chikungunya viruses. J Mol Diagn 17: 722-728, 2015. 